\title{
Breaststroke with Different Intensities Exercise on Cardiovascular Function and Blood Indexes of College Students
}

\author{
Min-gang Guo \\ Physical Education Department of Wuhan University of Technology, Hubei Province, China
}

\begin{abstract}
To explore the different exercise intensities breaststroke exercise on cardiovascular function, from exercise intensity angle to choose reasonable breaststroke intervention means in the improvement of cardiovascular function and provide a theoretical basis. Methods: randomly selected 60 samples, the control group routine life and learning, without any form of physical exercise, the experimental group for 18 weeks of different intensity exercise the breaststroke, each group weekly exercise two lessons, each lesson is 90 minutes, including the preparation, basic and relaxation part. Results: 1) HR, MSP and reduced significantly, SI, CI, MDP value rose somewhat, MAP values decreased slightly; 2) in the strength group SV elevated and very significant, HR, MSP, MDP, MAP decreased significantly, SI increased significantly; 3) large strength of group SV, SI increased and HR decreased, and were very significant, MSP, MDP, MAP decreased significantly. Conclusion: The different intensities of breaststroke exercise can have a positive impact on cardiovascular function and blood indices. In the strength group breaststroke exercise on cardiovascular function had the best effect.
\end{abstract}

Index Terms - Breaststroke; blood indexes; student

\section{Introduction}

Breaststroke is a mimic frog posture swimming of bionic sport, also a stroke is one of the oldest, slowest speed in the middle are four basic types of stroke, but its action and effort breath are both durable and suitable for long distance swimming. Breaststroke is one competitive stroke which is also a fashionable way of fitness; its value should not be overlooked. Since 1980s, domestic scholars on swimming of fitness value research gradually appears, Li Yinan [1], Zhong Xiting [2] Tang Yan [3], and other scholars research points out that swimming has a positive effect on adolescent physical and mental health, also scholars by animal experimental research thought swimming of value, as Dang Lilong in its different strength swimming training on large rat serum enzyme and the lipid of effect research under a text pointed out that general movement load in body movement metabolism function improve of adaptability changes, and beneficial to maintain health standards, movement and overload may be associated with exercise-induced injury of the body's tissues and organs, to the disadvantage of maintaining health [4]. But none of the results of existing research compares different exercise intensity fitness effects of swimming exercise on college students, and specifically scientific research on cardiovascular effects of a stroke on students' is blank in report. To fill in this gap, this paper takes breaststroke as an example, through the comparison of different exercise intensities breast exercises on cardiovascular function of college students, to swim breaststroke of course set up in colleges and universities and improve the teaching effect of providing experimental basis.

\section{Subjects and Method}

\section{A. Subjects}

From a randomly selected college freshmen of freshman medical examination table about 60 samples. For guarantee by object of with mass sexual, avoid potential of hybrid partial leaning, extraction standard is as follows: (1)No smoking, and alcohol, bad life habits; (2) Body health, no cardiovascular system disease and the other disease history; (3) At the age of 18 to 20 years, mean age $19.4 \pm 1.17$ age; (4) Sit-in bit blood pressure normal: contraction pressure $<140 \mathrm{mmHg}$, diastolic pressure <90mmHg; (5) Static income ECG check results normal. The subjects are randomly divided into the control group, low intensity group, in the strength group and large group, 15 people per group. In order to control the extraneous variables, error reduction, is conducted by the research group of the same teacher using breaststroke teaching. The teaching times from the September1, 2011 to January6, 2012.

\section{B. Experimental Methods}

\section{1) Sport Scheme}

In the entire process of experimental teaching, experimental conditions should be rigorously controlled experimental conditions. Control group (does not participation any form of exercise, $n=15)$; small strength Group (experimental-a group for 18 week movement load in $50 \% \sim 59 \%$ maximum heart rate of breaststroke exercises, $\mathrm{n}=15$ ); in the medium strength Group (experimental b group for 18 week movement load in 60\% 69\% maximum heart rate of breaststroke exercises, $n=15)$; large strength Group (experimental c group for 18 week movement load in $70 \% \sim 79 \%$ maximum heart rate of breaststroke exercises, $\mathrm{n}=15$ ), maximum heart rate $=220$-age. PolarA5 telemeter is used to monitor the heart rate in different exercise intensity, the experimental group is 90 minutes of instruction each time, exercise frequency is 3 times a week. Participants are tested under quiet conditions on an empty stomach in the morning, finally, test changes of cardiovascular system of selected college students, before and after the experiment.

\section{2 ) Testing Instrument and Indicator}

Test instruments including: XXG-E3-automatic, Omron 
HEM-7000 intelligent digital blood pressure monitor, cardiovascular function diagnosis instrument Italy SABA18selective channel volume Analyzer and LBY-N6C automatic blood rheology apparatus. Main test index including: HR, and SV, and SI, and MSP, and MDP, and MAP.

\section{3) Index Determination Method}

Using Omron HEM-7000 intelligent digital blood pressure monitor blood pressure, Japan ohm Dragon company; XXG-E3 cardiovascular function diagnosis instrument for automatic testing of cardiovascular function, by the Anhui Research Institute of electronic science, product standards Q/AAA013-1999.

\section{4) Data Statistics and Analysis}

Using SPSS13.0 of social statistical analysis software for statistical analysis of the experimental results, and find the values of indicators $( \pm)$, standard deviation $(\bar{x} \pm s)$, group differences between use independent samples $\mathrm{t}$ test, in the experimental group before and after difference use paired $\mathrm{T}$ test, $\mathrm{p}<0.05$ means significant difference, $\mathrm{p}<0.01$ says differences are very significant.

\section{Results and Discussion}

\section{A. Cardiac Function in Response to Different Intensities of Breaststroke Motion}

Compare the same index of three experimental groups and the control group, experimental A-group, HR fell apparent, $\mathrm{SV}$, and SI value rose apparently, and had significant differences $(\mathrm{p}<0.05)$; Experimental b Group HR fell very apparent, SV, SI value rose apparently, and had a significant difference $(\mathrm{p}<0.05)$ too, experimental $\mathrm{C}$-group HR fell rather dramatically, and had a very significant difference $(p<0.01)$, $\mathrm{SV}$, SI values rose significantly, significant differences $(p<0.05)$ too. After the experiment of three experimental groups, experimental A-group and C-Group HR, SV, SI value change apparently, and have significant differences $(\mathrm{p}<0.05)$, experimental group $\mathrm{b}$ and $\mathrm{c}$ groups in $\mathrm{HR}$, SV, SI value changes apparently, present significant differences $(\mathrm{p}<0.05)$.

Table 14 groups of subject experiment comparison of cardiac function before and after $(\bar{x} \pm s)$

\begin{tabular}{|c|c|c|c|c|}
\hline groups & time & $\mathrm{HR}(\mathrm{times} / \mathrm{min})$ & $\mathrm{SV}(\mathrm{ml} / \mathrm{b})$ & $\mathrm{SI}\left(\mathrm{ml} / \mathrm{b} / \mathrm{m}^{2}\right)$ \\
\hline \multirow{2}{*}{$\begin{array}{c}\text { Control } \\
\text { group }\end{array}$} & before & $71.12 \pm 7.23$ & $49.43 \pm 12.68$ & $47.36 \pm 10.46$ \\
\cline { 2 - 5 } & after & $72.43 \pm 7.02$ & $48.32 \pm 13.57$ & $46.29 \pm 9.34$ \\
\hline \multirow{2}{*}{$\begin{array}{c}\text { Group- } \\
\text { A }\end{array}$} & before & $73.57 \pm 6.92$ & $50.29 \pm 16.10$ & $49.75 \pm 11.63$ \\
\cline { 2 - 5 } & after & $64.10 \pm 8.27 * \prime$ & $62.59 \pm 19.47 * \prime$ & $52.71 \pm 9.87 '$ \\
\hline \multirow{2}{*}{$\begin{array}{c}\text { Group- } \\
\text { B }\end{array}$} & before & $73.12 \pm 8.34$ & $51.75 \pm 14.19$ & $50.65 \pm 12.44$ \\
\cline { 2 - 5 } & after & $56.46 \pm 8.64 * * "$ & $76.58 \pm 26.32 * * "$ & $61.14 \pm 10.37 * \prime$ \\
\hline \multirow{2}{*}{$\begin{array}{c}\text { Group- } \\
\text { C }\end{array}$} & before & $74.29 \pm 6.41$ & $56.52 \pm 12.29$ & $49.42 \pm 11.85$ \\
\cline { 2 - 5 } & after & $60.93 \pm 7.50 * * " 1$ & $70.75 \pm 17.62 * \prime$ & $54.20 \pm 9.52 * \prime$ \\
$\Delta$
\end{tabular}

Note: experimental test results before and after: $* \mathrm{P}<0.05$, ** $\mathrm{P}<0.01$; experimental group and control group with a test: $\mathrm{P}<0.05$; " $\mathrm{P}<$ $0.01 ;$ Compared with group-A: $\Delta \mathrm{P}<0.05 ; \Delta \Delta \mathrm{P}<0.01$; Compared with group-B: $\boldsymbol{\Delta} \mathrm{P}<0.05 ; \quad \boldsymbol{\Delta} \Delta \mathrm{P}<0.01$
B. Function of Vascular Response to Different Intensities of Breaststroke Motion

Three experimental comparison of results between experimental and control groups after the same group test, experiment a group of MSP, MDP, MAP, values are declining significantly, experimental group b MSP, MDP, falling on the MAP, experimental group c MSP, MDP, MAP.

After three experimental group experimental, experimental group a and $\mathrm{c}$ in the MSP, significant differences $(\mathrm{p}<0.05)$, experimental group $\mathrm{b}$ and group $\mathrm{c}$ in the MDP, MAP value change clear, significant differences $(\mathrm{p}<0.05)$.

After three sets of experimental group experimental, laboratory $b$ in MSP, MDP, MAP values fall on the most obvious, experimental group c second, experiment a group again. Indicate different strength breaststroke practice difference effect on vascular function in college students, with strength in best group, followed by high intensity group, group of small intensity again.

Table 24 set of subjects before and after the experimental vascular function $(\bar{x} \pm s)$

\begin{tabular}{|c|c|c|c|c|}
\hline groups & time & MSP $(\mathrm{kPa})$ & $\mathrm{MDP}(\mathrm{kPa})$ & MAP $(\mathrm{kPa})$ \\
\hline \multirow{2}{*}{$\begin{array}{c}\text { Control } \\
\text { group }\end{array}$} & before & $11.25 \pm 0.72$ & $8.92 \pm 0.58$ & $10.35 \pm 0.81$ \\
\cline { 2 - 5 } & after & $11.34 \pm 0.71$ & $9.03 \pm 0.49$ & $10.41 \pm 0.79$ \\
\hline \multirow{2}{*}{ Group-A } & before & $11.01 \pm 0.70$ & $8.36 \pm 0.77$ & $9.82 \pm 0.63$ \\
\cline { 2 - 5 } & after & $10.84 \pm 0.66 *{ }^{*}$ & $8.15 \pm 0.85^{\prime}$ & $9.51 \pm 0.68$ \\
\hline \multirow{2}{*}{ Group-B } & before & $11.49 \pm 0.32$ & $9.32 \pm 0.43$ & $10.47 \pm 0.64$ \\
\cline { 2 - 5 } & after & $10.44 \pm 0.70 * * "$ & $8.54 \pm 0.67 * * "$ & $9.42 \pm 0.75 * * "$ \\
\hline \multirow{2}{*}{ Group-C } & before & $10.52 \pm 0.93$ & $8.95 \pm 0.58$ & $10.04 \pm 1.15$ \\
\cline { 2 - 5 } & after & $10.24 \pm 1.13 * ' \Delta$ & $8.29 \pm 0.73 * \mathbf{\Delta}$ & $9.72 \pm 0.83 * ' \boldsymbol{\Delta}$ \\
\hline
\end{tabular}

\section{Recommendations and Conclusions}

Follow-up research should by select different age level of is try for object validation this research of conclusions, trying to full reveals different movement strength and different age structure crowd cardiovascular function and related blood index; Follow-up research also should concern swimming movement other constitute elements (as project type, and continued time) on different gender, and age structure crowd cardiovascular function and the other physiological, and psychological, Omni-directional of effect, for future reasonable select swimming project, Swimming place effective intervention programmers to provide theoretical support.

This study suggests that different exercise intensity of breast exercises can have positive effects on cardiovascular function of college students, but there is a difference. Group strength in breaststroke effects of exercise on the cardiovascular function of college students is the best, followed by high intensity group, group of small intensity again. Research results once again confirmed that aerobic exercise is an effective intervention to improve and enhance the body's cardiovascular function, strength and aerobic fitness and movement control exists. Study of school physical 
education teacher, for the future, such as sports, swimming and fitness enthusiasts make reasonable from the angle of exercise intensity swimming exercise prescription to provide practical support.

\section{References}

[1] Li Yinan. Swimming, aerobics on college students' physical and mental health. Journal of Beijing Sport University, 1999,22 (3): 86-89.
[2] Zhong Xiting. Swimming training on children's physical, morphology and function impact. Journal of Jinggangshan Medical College, 2008,15 (3): 74-75.

[3] Tang Yan. Swimming is the best choice for children's physical activity. Swimming, 2002 (2): 29

[4] Dang Lilong. Different intensity swimming training on serum enzymes and lipids. Journal of Northwest University (Natural Science Edition), 2007,37 (4): 610-612. 